\title{
sensors
}

ISSN 1424-8220

(C) 2006 by MDPI

http://www.mdpi.org/sensors

\section{High Frequency Quartz Micro Balances: A Promising Path to Enhanced Sensitivity of Gravimetric Sensors}

\author{
Chrisitan Kreutz $^{1,}{ }^{*}$, Jürgen Lörgen ${ }^{1}$, Boris Graewe ${ }^{1}$, Joachim Bargon ${ }^{1}$, Mayumi Yoshida ${ }^{2}$, \\ Zachary M. Fresco ${ }^{2}$ Jean M.J. Frèchet ${ }^{2}$ \\ ${ }^{1}$ Institute of Physical and Theoretical Chemistry, University of Bonn, 53115 Bonn, Germany \\ ${ }^{2}$ Department of Chemistry, University of California Berkeley, Berkeley, CA 94720-1460, USA \\ * Author to whom correspondence should be addressed. Email: kreutz@thch.uni-bonn.de
}

Received: 30 July 2005 / Accepted: 8 March 2006 / Published: 7 April 2006

\begin{abstract}
An array of $50 \mathrm{MHz}$ quartz microbalances (QMBs) coated with a dendronized polymer was used to detect small amounts of volatile organic compounds (VOCs) in the gas phase. The results were compared to those obtained with the commonly used $10 \mathrm{MHz}$ QMBs. The $50 \mathrm{MHz}$ QMBs proved to be a powerful tool for the detection of VOCs in the gas phase; therefore, they represent a promising alternative to the much more delicate surface acoustic wave devices (SAWs).
\end{abstract}

Keywords: Quartz micro balances, electronic noses, dendronized polymers, inverted mesa technology. 


\section{Introduction}

Since Sauerbrey's first successful attempt to weigh ultra-low masses using a quartz crystal microbalance (QMB) in 1969, many applications of this technology have been realised [1]. The recently launched Mars Mission Pathfinder, for example, which used the rover Sojourner to explore and characterise Mars, was equipped with an integrated QMB. There, it was used to measure how fast dust settles onto surfaces on Mars [2]. This quite extravagant application shall serve as an example for a plethora of others, including less exotic ones, where this kind of sensor is utilised today. Another recent application, which has entered the field-testing phase, is the realisation of an electronic nose that measures the concentration of Basamid ${ }^{\circledR}$, a herbicide produced by BASF, in the soil in agriculture. More precisely, the time dependence of the concentration of Basamid's decay product, methylisothiocyanate (MITC), has been measured and monitored successfully in more than 10 countries all over the world [3]. For applications like this one, the standard AT-cut QMBs have a number of practical advantages: They are robust, readily available in large amounts, inexpensive, and stable over a broad range of temperatures. Thus far, the fact that the fundamental resonance frequency of a quartz disk is inversely proportional to its thickness has prevented the use of frequencies $>20 \mathrm{MHz}$, since the quartz wafer becomes extremely fragile at thickness' corresponding to higher fundamental frequencies. Therefore, most applications of QMBs have utilised resonance frequencies centred on $10 \mathrm{MHz}$, which represents a good compromise between sensitivity and structural integrity. Nevertheless, higher basic frequencies in combination with good mechanical stability remain an attractive goal, since Sauerbrey's equation suggests an increase of the sensitivity of a QMB proportional to the square of the operating frequency. One possible, but infrequently used option to reach such higher frequencies is to excite a harmonic overtone using the same robust quartz as before. Yet, using a harmonic of the basic resonance frequency drastically reduces the stability of the QMB in leading to higher noise and thus poor performance.

Another approach uses a characteristically different kind of oscillator, which is based on utilising surface waves rather than volume oscillations. These so-called surface acoustic wave devices (SAWs) operate at frequencies ranging from $100 \mathrm{MHz}$ up to several GHz. Their drawbacks are their high sensibility towards changes in temperature and their increased demand towards the electronic circuitry required to operate the SAWs. Figure 1 shows the typical frequency range of the two types of electronic devices.

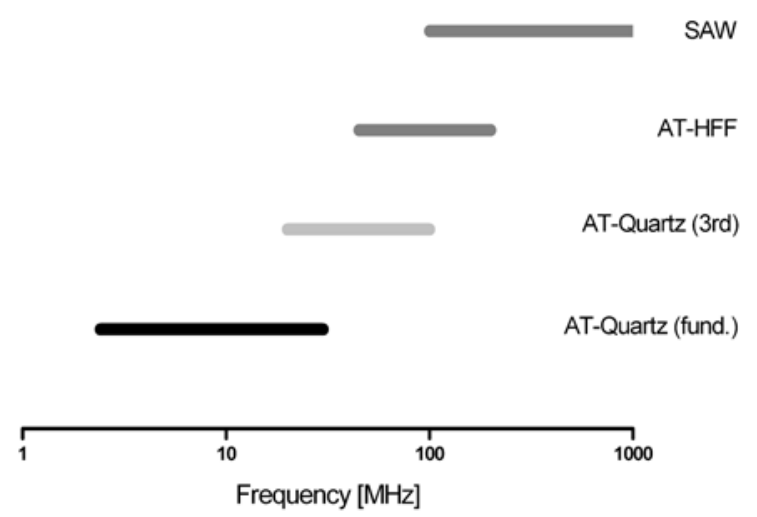

Figure 1. Typical frequency ranges of different types of gravimetric sensors based on oscillating quartzes [4]. 
A new approach to combine the benefits of higher frequencies of operation together with a high temperature tolerance is realised with so-called High Frequency Fundamental (= HFF-) Quartz crystals. Thus far, this fairly new type of resonator has predominantly been used for other types of high frequency technology, but only sparingly in connection with sensor applications [5]. Their higher frequency of operation in connection with their relatively good mechanical stability is realised by etching only a small circle in the middle of a regular $10 \mathrm{MHz}$ quartz crystals until it becomes so thin that its resonance frequency reaches $50 \mathrm{MHz}$ or even higher frequencies. Consequently, only this thin inner circle is excited to an oscillation via an electrode. The mechanical stability of this design of a quartz resonator is due to the remaining thick outer ring (Figure 2).

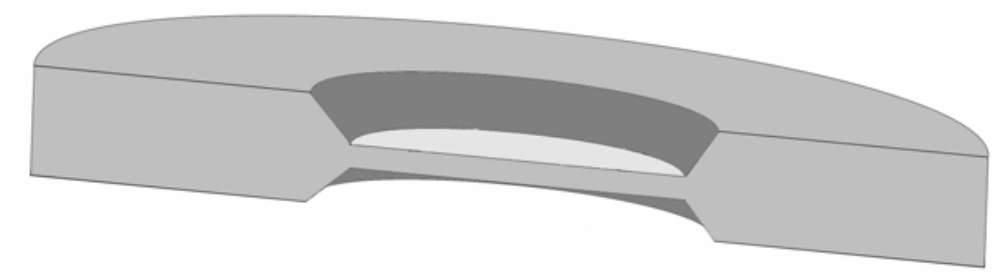

Figure 2. Cross section of an HFF quartz wafer realised with the Inverted Mesa Technology.

This production technique is called "Inverted Mesa Technology“. In this fashion, quartz crystals with basic frequencies up to $200 \mathrm{MHz}$ can be realised. The HFF-quartzes used in this study were obtained from KVG International GmbH, Neckarsulm, Germany, with a basic resonance of $51.84 \mathrm{MHz}$ and a HC52 mount. The same company delivered the $10 \mathrm{MHz}$ quartz crystals used as reference.

Below the results of first measurements concerning the detection of volatile organic compounds (VOCs) obtained with coated HFF as well as with $10 \mathrm{MHz}$ quartzes are presented and compared.

\section{Experimental set-up}

Well-defined mixtures of nitrogen and a variety of gaseous analytes are fed into the temperaturecontrolled measuring cells via a gas-mixing unit containing up to 24 QMBs coated with a variety of dendritic polymers. During a measurement cycle the surfaces of the sensors, which are coated with a sensor-active host material, are exposed to different concentrations of an individual analyte. A personal computer (C) controls both the data acquisition and hence, the individual QMB frequencies and the temperature.

Each individual QMB is prepared from an AT-cut quartz crystal operating at a resonance frequency of either 51.84 or $10 \mathrm{MHz}$. Both sides of the quartz surface are coated and contacted with aluminiumplated electrodes $(51.84 \mathrm{MHz})$ or gold-plated electrodes $(10 \mathrm{MHz})$, but only one is coated with a sensoractive substance (i.e., a sensor-active host compound). Due to passivation, the use of aluminium as the electrode material is opportune. However, only small and even slow variances ( 2.5\%) were noticed during our experiments, which are tolerable in case of measuring only relative frequency changes.

The coating of the QMBs surfaces was achieved via the electrostatic spray method, a method well known from mass spectrometry [6,7]. For this purpose, the host compounds were dissolved in a polar 
solvent (e.g., THF) and transferred into a Hamilton syringe. The spray was aerosolized and accelerated via an applied high voltage DC (2 - $3 \mathrm{kV})$, which was applied to the top electrode of the QMBs. As the solvent evaporates on its way from the tip of the syringe to the quartz surface, the host compound forms a homogeneous layer with almost no intercalated solvent. Using in-situ monitoring of the QMBs oscillation frequency during the coating process the increasing layer thickness can easily be followed in terms of a frequency shift, which allows the fabrication of a reproducible coating thickness.

When the so-coated QMBs are exposed to volatile analytes, the adsorption and/or inclusion of these guests into/at the host's volume or surface causes the effective oscillating mass of the QMB to increase, which results in a decrease of the resonance frequency. The magnitude of this change of the oscillating frequency is a measure of the amount of the included analyte, and the kinetics is a measure of the host - guest interaction. All observed sensor responses were reversible, ensuring that the binding is noncovalent.

With this setup we have exposed several QMBs coated with host compounds to different VOC`s at $30{ }^{\circ} \mathrm{C}$, and tested the performance of quartz crystals operating either at 10 or $51.84 \mathrm{MHz}$.

After each exposure, the cell was purged thoroughly with pure nitrogen in order to regenerate the selective layer by desorption of the guest molecules.

\section{Results and discussion}

Comparisons were conducted between quartz crystals with different basic frequencies but identical sensor-active coating substances. Figure 3 outlines the result obtained with regard to the $10 \mathrm{MHz}$ and $51.84 \mathrm{MHz}$ quartzes. As coating material a functionalized polymer was used, which had previously been tested to be especially suitable for the detection of electron-poor aromatics in preceding screening tests. More specifically poly(p-hydroxystyrene), which has been dendronized to G1 using benzylidine protected bishydroxymethypropionic anhydride [8] (Figure 3). The VOCs tested were the electrondeficient aromatics 3- and 4-nitrotoluene.



Figure 3. Dendronized polymer with different generations. 
Figure 4 shows the 11.5 -fold increase in signal intensity that is attributable to the higher basic frequency of the resonators. Despite this remarkable amplification this value is below the expected result according to Sauerbrey's equation. According to this equation, an about 36-fold amplification in sensitivity would have been expected.

This effect can be explained considering the viscoelastic properties of the coating. The latter are unaccounted for in Sauerbrey's equation. Raising the fundamental frequency from $10 \mathrm{MHz}$ to $51.84 \mathrm{MHz}$ one experiences a nonlinear influence of the viscoelastic properties as already had been shown in detail elsewhere [9]. A detailed treatment of both the theoretical background and exact calculations is way beyond the scope of this work and thus, may be derived with the help of the above cited reference.

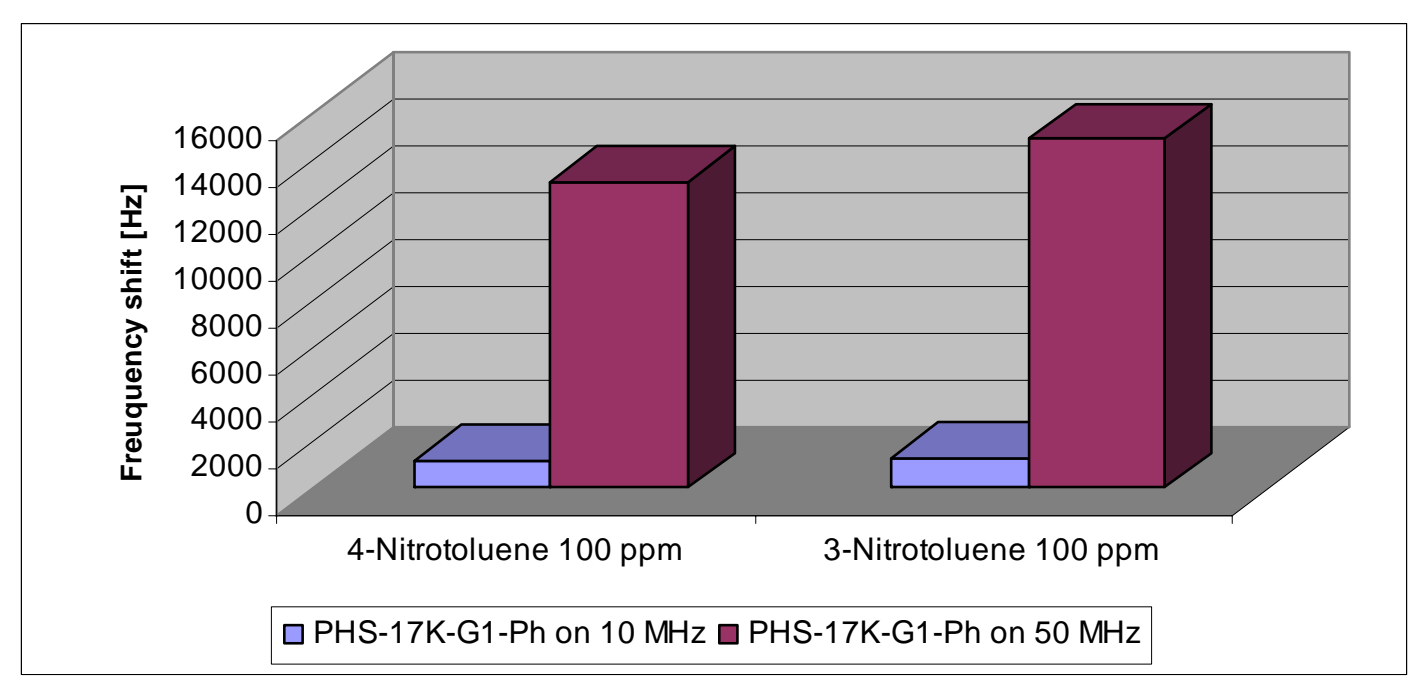

Figure 4. Frequency shift measured with 10 and $50 \mathrm{MHz}$ QMBs.

To assess any aging of the coatings, measurements have been conducted during a period of two months. Within this span of time the depletion of the coated quartzes' fundamental frequencies never exceeded more than $2.5 \%$. Thus, a degradation of the coating substances is very slow if at all significant.

\section{Conclusion}

Despite the fact that the theoretically extrapolated signal amplification was not realized as expected based on the higher oscillating frequency of $51,84 \mathrm{MHz}$, the superior performance and strikingly higher detection limit of these HFF-QMBs has successfully been demonstrated. Our results are especially promising for the detection of trace amounts of VOCs in the gas phase. Therefore, the availability of HFF quartzes can be expected to boost and broaden the application spectrum of gravimetric sensors. Additional measurements using different analytes for the comparison of the two QMBs will be made soon.

\section{References}

1. Sauerbrey, G. Verwendung von Schwingquarzen zur Wägung dünner Schichten und zur Mikrowägung. Z. Phys., 1959, 155, 206. 
2. http://www.grc.nasa.gov/WWW/OptInstr/mars.html, October 2004.

3. Patent EP 1370862 B1: Method and apparatus for the detection of methyl isothionate in air samples, 28.02.2002.

4. http://www.kvg-gmbh.de.

5. Ward, D.; Lin, Z.; Yip, C.; Joseph, S. Operation of an ultrasensitive 30-MHz quartz crystal microbalance in liquids. Anal. Chem., 1993, 65, 1546.

6. Taylor, G.I. Disintegration of water drops in an electric field. Proc. R. Soc. London Ser. A, 1964, 280, 383.

7. Fenn, J.B. Elektrospray: Molekulare Elefanten lernen das Fliegen. Angew. Chem., 2003, 115, 3999.

8. Grayson, S.M.; Frechet, J.M.J. Divergent synthesis of dendronized poly(p-hydroxystyrene). Macromolecules, 2001, 34, 6542.

9. Uttenthaler, E.J. Hochempfindliche akustische Sensorelemente für die Flüssigkeits- und Biosensorik. ISBN 3831601321, Herbert Utz Verlag, München, 2002.

(C) 2006 by MDPI (http://www.mdpi.org). Reproduction is permitted for non-commercial purposes. 